\title{
INTEGRAL RING EXTENSIONS AND PRIME IDEALS OF INFINITE RANK
}

\author{
WILLIAM HEINZER
}

Abstract. An example is constructed showing that for an integral ring extension $R \subset T$, and a prime ideal $P$ of $R$ having infinite rank, it can happen that in $T$ each prime ideal lying over $P$ has finite rank.

By the rank (or height) of a prime ideal $P$ in a commutative ring $R$ is meant the maximal length of descending chains of prime ideals of $R$ starting with $P$. Thus $P$ has rank $n$ if there exists a descending chain $P=P_{0} \supset P_{1} \supset \cdots \supset P_{n}$, but no such chain of longer length; and $P$ has infinite rank (or rank $\infty$ ) if there exist arbitrarily long chains of primes descending from $P$. Let $R \subset T$ be a pair of commutative rings (having a common identity). One says that the going up property (GU) holds for the pair $R \subset T$ if whenever $P \subset P_{0}$ are prime ideals in $R$ and $Q$ is a prime of $T$ such that $Q \cap R=P$, then there exists a prime $Q_{0}$ in $T$ such that $Q \subset Q_{0}$ and $Q_{0} \cap R=P_{0}$. It is well known that if $T$ is in tegral over $R$, then GU holds for the pair $R \subset T$; and it can be readily seen that if $R \subset T$ satisfies GU and $P$ is a prime ideal in $R$ of rank $n$, then there exists in $T$ a prime ideal $Q$ such that $Q$ has rank $\geqq n$ and $Q \cap R=P$ [3, Theorem 46, p. 31]. We show, however, that this result cannot be extended to primes of rank $\infty$ even for $R$ an integral domain and $T$ the integral closure of $R$. Of course GU insures that there can be no fixed bound on the ranks of the primes of $T$ lying over a rank $\infty$ prime $P$ of $R$. Thus in our example there must be infinitely many primes of $T$ lying over $P$. In particular, $T$ cannot be a finite $R$ module $[1, \mathrm{p} .40]$.

The idea involved in our construction may be stated as follows.

Lemмa. Let $R$ be a quasi-local domain with maximal ideal $P$ and quotient field $K$. Assume that for each positive integer $n$ there exists a valuation ring of $K$ containing $R$ and having rank $n$, but that $R$ is contained in no valuation ring of $K$ having infinite rank. Let $T$ be the integral closure of $R$. If $T$ is a Prïfer domain, then $P$ has infinite rank but each prime ideal of $T$ has finite rank.

Proof. If $Q$ is a prime ideal of $T$, then the localization $T_{Q}$ is a

Received by the editors June 23, 1970.

AMS 1969 subject classifications. Primary 1320.

Key words and phrases. Integral ring extension, prime ideal, going up property, valuation ring, Prüfer domain. 
valuation ring and the rank of the prime ideal $Q$ equals the rank of the valuation ring $T_{Q}$. Thus each prime of $T$ has finite rank and by intersecting chains of primes of $T$ with $R$, we see that $P$ has infinite rank.

CONSTRUCTION OF THE EXAMPLE. Let $k$ be an arbitrary field and let $\left\{x_{i}\right\}_{i=1}^{\infty}$ be a collection of indeterminates over $k$. We construct a rank one valuation ring $V_{1}$ on the field $K=k\left(x_{1}, x_{2}, \cdots\right)$ such that $V_{1}$ has the form $k+M_{1}$ where $M_{1}$ is the maximal ideal of $V_{1}$. This can be done, for example, by mapping the $x_{i}$ onto rationally independent real numbers and then extending this map to a valuation of $K$ trivial on $k$. The $x_{i}$ having rationally independent values assures that $k$ maps isomorphically onto the residue field of $V_{1}$ and hence that $V_{1}=k+M_{1}$. For each integer $n \geqq 2$, let $L_{n}$ denote the field $k\left(\left\{x_{i} \mid i \leqq n\right.\right.$ or $\left.\left.i \geqq 2 n\right\}\right)$. Thus $K=L_{n}\left(x_{n+1}, \cdots, x_{2 n-1}\right)$ and $x_{n+1}, \cdots$, $x_{2 n-1}$ are algebraically independent over $L_{n}$. Consider the valuation ring $V_{1} \cap L_{n}$. By mapping $x_{n+1}, \cdots, x_{2 n-1}$ onto suitably chosen elements of a suitable totally ordered abelian group containing the value group of $V_{n}$ we can obtain a valuation ring $V_{n}$ of $K$ such that:

(1) $V_{n} \cap L_{n}=V_{1} \cap L_{n}$.

(2) $V_{n}$ has rank $n$.

(3) $V_{n}$ has the form $k+M_{n}$ where $M_{n}$ is the maximal ideal of $V_{n}$. See, for example, [1, Proposition 1, p. 161].

Let $P=\bigcap_{i=1}^{\infty} M_{i}$ and let $R=k+P$. We note that $R$ is a quasi-local domain with maximal ideal $P$. For if $\alpha$ is a nonzero element of $k$ and $m \in P$, then $(\alpha+m)^{-1}=\alpha^{-1}+m^{\prime}$, where $m^{\prime}=-m / \alpha(\alpha+m) \in M_{i}$ for each $i$, so $m^{\prime} \in P$. Let $T$ be the integral closure of $R$.

Claim. $T$ is a Prüfer domain with quotient field $K, T=\bigcap_{i=1}^{\infty} V_{i}$, and no valuation ring between $T$ and $K$ has infinite rank.

Proof. Let $K_{n}=k\left(x_{1}, \cdots, x_{n}\right), R_{n}=R \cap K_{n}$, and let $T_{n}$ be the integral closure of $R_{n}$. Note that for $s \geqq n, V_{s} \cap K_{n}=V_{1} \cap K_{n}$. Hence

$$
R_{n}=k+\left(\bigcap_{i=1}^{n-1} M_{i} \cap K_{n}\right)
$$

We show that $T_{n}=\bigcap_{i=1}^{n-1} V_{i} \cap K_{n}$. If $y \in \bigcap_{i=1}^{n-1} V_{i} \cap K_{n}$ then there exists $a_{i} \in k$ such that $y-a_{i} \in M_{i}$, for each $i$ such that $1 \leqq i<n$. It follows that $\prod_{i=1}^{n-1}\left(y-a_{i}\right) \in \bigcap_{i=1}^{n-1} M_{i} \cap K_{n} \subset R_{n}$ so $y$ satisfies an equation of integral dependence over $R_{n}$. Thus $T_{n}$ is a finite intersection of valuation rings of the field $K_{n}$. Hence $T_{n}$ is a Prüfer domain with quotient field $K_{n}$ and each valuation ring containing $T_{n}$ contains some $V_{i} \cap K_{n}\left[1\right.$, p. 132-134]. It follows that $T=\bigcup_{i=1}^{\infty} T_{i}$ is also Prüfer [2, p. 260], $T$ has quotient field $K$, and $T=\bigcap_{i=1}^{\infty} V_{i}$. Now 
suppose $W$ is a valuation ring between $T$ and $K$. Since $W$ contains $T_{n}, W$ contains some $V_{i} \cap K_{n}$. If $W$ contains $V_{1} \cap K_{n}$ for all $n$, then $W$ contains $V_{1}$ so either $W=V_{1}$ or $W=K$. If $V_{1} \cap K_{n} \varangle W$, then for $s \geqq n$, let $W_{s}=W \cap K_{s}$. We know that $V_{j} \cap K_{s} \subset W_{s}$ for some $j<s$. But, for $j \geqq n, V_{j} \cap K_{8} \cap K_{n}=V_{1} \cap K_{n}$, so $W_{8}$ is contained in $V_{j} \cap K_{8}$ for some $j<n$. Since $V_{j}$ has rank $j$, we see that $W_{s}$ has rank $<n$. It follows that $W=\bigcup_{s=n}^{\infty} W_{s}$ also has rank less than $n$.

\section{REFERENCES}

1. N. Bourbaki, Algèbre commutative, Chapitres 5, 6, Actualités Sci. Indust., no. 1308, Hermann, Paris, 1964. MR 33 \#2660.

2. R. Gilmer, Multiplicative ideal theory, Queen's Papers in Pure and Appl. Math., no. 12, Queen's University, Kingston, Ont., 1968. MR 37 \#5198.

3. I. Kaplansky, Commutative rings, Allyn and Bacon, Boston, Mass., 1970.

Louisiana State University, Baton Rouge, Louisiana 70803

Purdue University, LafayetTe, Indiana 47907 